\title{
A collision avoidance strategy for a potential natural satellite around the asteroid Bennu for the OSIRIS-REx mission
}

\author{
Alinda K. Mashiku* and J. Russell Carpenter ${ }^{\dagger}$ \\ NASA Goddard Space Flight Center, Greenbelt, MD, 20771, USA
}

\begin{abstract}
The cadence of proximity operations for the OSIRIS-REx mission may have an extra induced challenge given the potential of the detection of a natural satellite orbiting the asteroid Bennu. Current ground radar observations for object detection orbiting Bennu show no found objects within bounds of specific size and rotation rates. If a natural satellite is detected during approach, a different proximity operation cadence will need to be implemented as well as a collison avoidance strategy for mission success. A collision avoidance strategy will be analyzed using the Wald Sequential Probability Ratio Test.
\end{abstract}

\section{Introduction}

7 HE Origins Spectral Interpretation Resource Identification Security Regolith EXplorer (OSIRIS-REx) 1 mission is a NASA near-earth asteroid (NEA) sample return mission to the asteroid Bennu (also known as 1999 RQ36). OSIRIS-REx is set to launch in late 2016 and will spend about two years in cruise towards Bennu, a period of time performing proximity operations and finally a capsule sample return back to Earth in 2023. The asteroid Bennu is currently the most accessible carbonaceous asteroid and potentially harzardous asteroid due to its predicted probability of impacting the Earth relative to other asteroids in the future. ${ }^{1}$

Bennu has a rapid rotation rate of about 4.29 hrs for a Bennu sidereal day. ${ }^{2}$ Most NEAs of spheroidal shapes and rapid rotation rates have been found to be primaries of a binary system. A binary system is when two objects rotate about a barycenter (the center of mass is contained in neither object). About $16 \%$ of NEAs with diameters larger than $200 \mathrm{~m}$ may belong to binary systems. ${ }^{3}$ Therefore, there is a statistical chance that the asteroid Bennu may have a natural satellite orbiting about itself. Ground-based radar observations of Bennu in 1999 and 2005 ruled out the possibility of natural satellites with specific diameters and rotation rates (Please see Table 1).

Table 1. Current radar results showing characteristics of satellites not detected ${ }^{2}$

\begin{tabular}{|c|c|}
\hline Natural Satellites Sizes Not Detected & Rotation Rates \\
\hline Diameter $>15 \mathrm{~m}$ & $1-\mathrm{min}$ \\
Diameter $>5 \mathrm{~m}$ & $1-\mathrm{hr}$ \\
Diameter $>2 \mathrm{~m}$ & $24-\mathrm{hr}$ \\
\hline
\end{tabular}

However, it's been discovered that some binary systems have primaries spinning with much faster rates than Bennu. ${ }^{2}$ The rotation rates of an asteroid determine whether the conditions are feasible for the asteroid to be a part of a binary system or have a natural satellite rotating about its own central body. The recent observations between 1999 and 2005 have also not detected any change in the rotation rate nor could we accurately predict a change in the rotation rate from these observations. ${ }^{2}$ Bennu is classified as an Apollo asteroid. Apollo asteroids are Earth crossing NEAs with semi-major axes larger than 1 AU and a perihelion distance less than Earth's aphelion (1.017 AU). As of recent, it has been detected that there are 34 Apollo asteroids with moons or that could be classified as a primary in a binary system. ${ }^{4}$ There are three asteroids whose relative sizes are in comparison to Bennu as listed in Table 2.

\footnotetext{
*Aerospace Engineer, Navigation and Mission Design Branch, and AIAA Member.

${ }^{\dagger}$ Deputy Project Manager/Technical, Space Science Mission Operations Project, Associate Fellow, AIAA
} 
Table 2. Known Apollo Asteroids with a Natural Satellite (Moon)

\begin{tabular}{|c|c|c|c|c|}
\hline Name of Asteroid & Diameter $(\mathrm{km})$ & Name of Moon & Diameter $(\mathrm{km})$ & Separation $(\mathrm{km})$ \\
\hline 1999 DJ4 & $0.43 \pm 0.08$ & $\mathrm{~S} / 2004$ & $0.21 \pm 0.05$ & 0.8 \\
2002 AM31 & $0.45 \pm 0.05$ & $\mathrm{~S} / 2012$ & 0.11 & 1.5 \\
2004 DC & 0.36 & $\mathrm{~S} / 2006$ & $0.21 \pm 0.07$ & $0.75 \pm 0.045$ \\
\hline
\end{tabular}

This warrants a need for a significant collision avoidance strategy for successful proximity operations and overall mission success. The OSIRIS-REx mission is a complex mission that is composed of several mission phases from launch to sample return and all the intricate proximity operation phases in between. Therefore, during approach, it has been predetermined that a natural satellite search campaign would be implemented to search for, characterize and generate an ephemeris of any potential natural satellite(s). It is the task of the Flight Dynamics team to determine a method to predict the natural satellite(s), develop a realistic cadence of obtaining tracking measurements, and define a collision avoidance strategy.

The potential of detecting natural satellites around Bennu could be evaluated in two ways: (1) Based on current radar observations for size and spin rates; (2) Based on discovered Apollo asteroids of similar size compared to Bennu. The focal point for this preliminary study will center on a natural satellite of a much smaller size based on what the recent observations around Bennu were not able to detect as itemized in Table 1. Most carbonaceous chondritic meteorites like Bennu have densities falling within the range of $2-3 \mathrm{~g} / \mathrm{cm}^{3}$ and some even up to $4 \mathrm{~g} / \mathrm{cm}^{3} .{ }^{5}$ A sample natural satellite is assumed with a density of $3.3 \mathrm{~g} / \mathrm{cm}^{3}$ and a mass of $2500 \mathrm{~kg}$. The cross sectional area is approximately $1 \mathrm{~m}^{2}$ with a radius of $0.75 \mathrm{~m}$.

Most of the crucial proximity operations of the OSIRIS-REx mission will take place in a terminator orbit. A terminator orbit's plane is normal to the sun vector. Since the solar radiation pressure exerts significant perturbations on spacecraft orbiting Bennu, the terminator orbit is a realm of relative orbit stability for proximity operations. Under those circumstances, it is also the orbit in which a natural satellite would potentially reside if one were to exist.

In this preliminary analysis, we simulate a natural satellite orbit trajectory in the terminator plane slightly off-phase from the OSIRIS-REx spacecraft. Our goal is to develop a decision making scheme on whether or not to perform a collision avoidance maneuver and at what probabilities will we encounter false alarm rates and missed detections given a subset of measurements and assumed apriori probabilities.

\section{Collision Avoidance Approaches}

In space operations, whether it be in Low Earth Orbit (LEO) or interplanetary trajectories, collision avoidance strategies are typically implemented to enable safe and successful completion of missions. With the increase in space debris in LEO, numerous approaches have been studied and implemented to maintain the safe operations of space assets by determining whether a maneuver is necessary or not, given certain deciding parameters. The collision probability as a function of time, has been widely used as a deciding parameter on whether a maneuver is necessary or not.

In regions with a high density of space debris (such as LEO), Kessler et al. ${ }^{6}$ developed a flux model to statistically model the orbital debris environment. Based on the debris population tracked by the United States Space Command (USSPACECOM), ground telescope data and measurements of debris impacts on satellites, the model predicts the number of debris or objects passing through a unit area (flux) as a function of time. The collision probability is computed from the Kessler model due to the flux of all objects, tracked and untracked and those that are larger than $10 \mathrm{~cm} .{ }^{7}$ In 2012, the International Space Station (ISS) had a record number of four collision threats, where the masses of these objects had the capability to inflict serious damage to the ISS in the event of a collision. ${ }^{7}$ If a predicted conjunction was to yield a probability of collision of 1 in 10,000, then a collision avoidance maneuver by the ISS is to be executed unless the maneuver would lead to an even greater risk to the ISS or its crew. ${ }^{6-8}$

With the availability of a calculated collision probability, collision avoidance decision making may be implemented based on whether the collision probability exceeds a given threshold or not. However, if a maneuver is implemented based on a singular metric, the decision involved does not incorporate other probabilistic information such as the false alarm and the missed detection. ${ }^{8}$ In the event of a false alarm, the collision probability might have met or exceeded the decision-making threshold and an unnecessary maneuver 
would have been implemented that could have been possibly avoided, hence saving fuel and time. On the other hand, in the event of a missed detection, the costs are much higher than those of the false alarm, in which a much needed maneuver is not implemented and the event could result in a collision.

Other work involving computing collision probability have delved into using the calculated relative state of the space objects at the time of closest approach (TCA). A Monte Carlo method is used to generate a mean and covariance of the relative state that can be used to provide the probability density and hence determine the collision probability. ${ }^{6-9}$

The Systems Tool Kit (STK) Collision Avoidance Tool (CAT) implements several algorithms in calculating the collision probability along with other metrics such as the approach angle, relative speeds and the time of closest approach. ${ }^{10}$ In this paper, we implement the Wald Sequential Probability Ratio Test (WSPRT) that uses sequential state estimates of the collision probability to enable decision making processes beyond a singular collision probability threshold method. The WSPRT uses a prior determination of the collision risk that does not involve any measurements, and includes the acceptable false alarm and missed detection rates that are scenario or mission specific. With available successive measurement updates, the WSPRT provides a decision making parameter based on the Likelihood ratio. This implementation has been well developed for the Magnetospheric Multi-Scale (MMS) mission, as detailed in references. ${ }^{8,11}$ Therefore, in this application for the OSIRIS-REx mission, a prior collision risk probability needs to be determined as well as a mission-acceptable false alarm and missed detection rate. For this paper, we will demonstrate various values of these parameters and how they affect the decision making process.

\section{Wald Sequential Probability Ratio test and the Likelihood ratio}

For two space objects whose position and velocity vectors are random variables at a given time $t$, the true miss distance at the time of closest approach $t_{c a}$ can be denoted as $r_{*}=r_{c a}$. Given a set of observations at times $t_{k}$ prior to $t_{*}=t_{c a}$, the set of observations (collection of realized random variables) can be denoted as, $\mathbb{Y}_{k}=\left\{y_{k}, y_{k-1}, \ldots, y_{1}\right\}$. The joint density is defined as $p_{\mathbf{Y}_{\mathbf{k}}}\left(y_{k}, y_{k-1}, \ldots, y_{1}\right)=p_{\mathbf{Y}_{\mathbf{k}}}\left(\mathbb{Y}_{k}\right)$.

We define a hardbody radius of $\mathbb{R}$, such that if $\left\|r_{*}\right\| \leq \mathbb{R}$, then the true miss distance is unsafe and is categorized by the null hypothesis $\mathcal{H}_{0}$. Conversely, the alternate hypothesis $\mathcal{H}_{1}$ applies if $\left\|r_{*}\right\| \geq \mathbb{R}$, such that the true miss distance is safe. The likelihood ratio is then calculated as follows:

$$
\begin{aligned}
\Lambda_{k} & =\frac{p\left(\mathbb{Y}_{k} \mid \mathcal{H}_{1}\right)}{p\left(\mathbb{Y}_{k} \mid \mathcal{H}_{0}\right)} \\
& =\frac{p\left(\mathbb{Y}_{k}|| \mid r_{*} \| \geq \mathbb{R}\right)}{p\left(\mathbb{Y}_{k}||\left|r_{*}\right| \leq \mathbb{R}\right)}
\end{aligned}
$$
as: $^{12}$

If the set $\mathbb{B}$, contains all the states that will result in a collision, the conditional probabilities are calculated

$$
\begin{aligned}
p\left(\mathbb{Y}_{k} \mid\left\|r_{*}\right\| \in \mathbb{B}\right)= & \frac{\prod_{i=1}^{k}\left(\frac{1}{(2 \pi)^{\frac{n}{2}} \sqrt{\left|\hat{P}_{* \mid i}\right|}}\right) \sqrt{\frac{\left|\hat{P}_{*|k|}\right|}{\left|\hat{P}_{*|0|}\right|}} e^{-\frac{1}{2} \alpha} P_{c \mid k}}{P_{c \mid 0}} \\
p\left(\mathbb{Y}_{k} \mid\left\|r_{*}\right\| \notin \mathbb{B}\right)= & \frac{\prod_{i=1}^{k}\left(\frac{1}{(2 \pi)^{\frac{n}{2}} \sqrt{\left|\hat{P}_{* \mid i}\right|}}\right) \sqrt{\frac{\left|\hat{P}_{*|k|}\right|}{\left|\hat{P}_{*|0|}\right|}} e^{-\frac{1}{2} \alpha}\left(1-P_{c \mid k}\right)}{1-P_{c \mid 0}}
\end{aligned}
$$

where

$$
\begin{aligned}
P_{c \mid k} & =\frac{1}{\left(2 \pi^{\frac{n}{2}} \sqrt{\mid \hat{P}_{* \mid k}}\right)} \int_{\mathbb{B}} \exp \left(-\frac{1}{2}\left(\hat{r}_{* \mid k}-r_{*}\right)^{T} \hat{P}_{* \mid k}^{-1}\left(\hat{r}_{* \mid k}-r_{*}\right)\right) d r_{*} \\
P_{c \mid 0} & =\frac{1}{\left(2 \pi^{\frac{n}{2}} \sqrt{\mid \hat{P}_{* \mid 0}}\right)} \int_{\mathbb{B}} \exp \left(-\frac{1}{2}\left(\hat{r}_{*}-r_{* \mid 0}\right)^{T} \hat{P}_{* \mid 0}^{-1}\left(r_{*}-\hat{r}_{* \mid 0}\right)\right) d r_{*}
\end{aligned}
$$

$P_{c \mid 0}$ is calculated based on the apriori probability of collision and $P_{c \mid k}$ is calculated conditioned on the available measurements to determine the probability of collision at time $t_{c a}$. 
The likelihood ratio $\Lambda_{k}$ at a given time $k$, guides the decision based on false alarms and missed detection criteria. Given a probability of a false alarm $P_{f a}$ and probability of a missed detection $P_{m d}$, then limits A and $\mathrm{B}$ are defined;

$$
\frac{1-P_{f a}}{P_{m d}} \geq A \quad \text { and } \quad \frac{P_{f a}}{1-P_{m d}} \leq B
$$

Generally, there exist no analytical methods to determine the $P_{f a}$ and $P_{m d}$ rates and so Wald suggests the use of a targeted $\bar{P}_{f a}$ and targeted $\bar{P}_{m d .}{ }^{13}$ This in turn redefines the limits A and B as follows:

$$
A=\frac{1-\bar{P}_{f a}}{\bar{P}_{m d}} \quad \text { and } \quad B=\frac{\bar{P}_{f a}}{1-\bar{P}_{m d}}
$$

If $B<\Lambda_{k}<A$, the ratio test would suggest to continue seeking additional observations and hence the decision is inconclusive. Otherwise, if $\Lambda_{k} \leq B$ then you would accept the null hypothesis and maneuver, and if $\Lambda_{k} \geq A$ you would accept the alternate hypothesis.

Therefore, the likelihood ratio can be expressed as:

$$
\begin{aligned}
\Lambda_{k} & =\frac{p\left(\mathbb{Y}_{k}||\left|r_{*}\right| \mid \notin \mathbb{B}\right)}{p\left(\mathbb{Y}_{k}||\left|r_{*}\right| \mid \in \mathbb{B}\right)} \\
& =\frac{1-P_{c \mid k}}{P_{c \mid k}} \frac{P_{c \mid 0}}{1-P_{c \mid 0}}
\end{aligned}
$$

\section{Scenario Examples}

In this section we will look at a simulated example that models a natural satellite orbiting the asteroid Bennu on a potential collision course with the OSIRIS-REx spacecraft. For most of the proximity operations around Bennu, the most stable orbit is the terminator orbit that lies in a perpendicular plane to the Sun's direction. Since the asteroid is relatively small in size $(\approx 500 \mathrm{~m}$ in diameter $)$, the solar radiation pressure is one of the largest perturbations around Bennu. Therefore, one could assume that if a natural satellite were to exist in a stable orbit, it would lie in the terminator plane as well. Similarly, in reference to the information given in Table 2, the separation distances of the natural satellites/moons to the asteroids are in a similar order of where we are planning the OSIRIS-REx's proximity operations to take place.

In this example, we would look at two scenarios. For each scenario, we simulate range, azimuth and elevation measurements of the natural satellite from the spacecraft to provide state updates at the cadence of the measurements. The measurements are taken at a 60 second time-step and after every hour the ephemeris is predicted to the time of closest approach to determine the probability of collision, given the targeted false alarm and targeted missed detection rates. The measurement noise variance for the range, azimuth and elevation used are $\left(1 \times 10^{-5}\right)^{2} \mathrm{~km}^{2},\left(1 \times 10^{-3}\right)^{2} \mathrm{rad}^{2}$, and $\left(1 \times 10^{-3}\right)^{2} \mathrm{rad}^{2}$ respectively. A hardbody radius of 10 meters was selected for this analysis and an apriori state covariance with position and velocity variances of $(2 / 3 * \mathbb{R})^{2} \mathrm{~km}^{2}$ and $\left(1 \times 10^{-6}\right)^{2}(\mathrm{~km} / \mathrm{s})^{2}$ respectively. The initial state vectors are defined at the epoch 18 Feb 2019 00:00:00.000 UTC. For this preliminary study, a 500 case Monte Carlo run is implemented in the analysis of the following scenarios.

Since the decision to maneuver is recommended when $\Lambda_{k} \leq B$ and the conjunction is rendered safe if $\Lambda_{k}>A$, the decision thresholds can be written as: ${ }^{8}$

$$
\begin{aligned}
P_{c \mid k} & \geq \frac{P_{c \mid 0}}{B+(1-B) P_{c \mid 0}}=P_{c}^{\text {Alarm }} \\
P_{c \mid k} & <\frac{P_{c \mid 0}}{A+(1-A) P_{c \mid 0}}=P_{c}^{\text {Dismiss }} \\
\text { where } \quad P_{c \mid k} & =\frac{P_{c \mid 0}}{\Lambda_{k}+\left(1-\Lambda_{k}\right) P_{c \mid 0}}
\end{aligned}
$$

where A and B are defined in Equation 8. We then observe where the $P_{c \mid k}$ values lie with respect to $P_{c}^{\text {Alarm }}$ and $P_{c}^{\text {Dismiss }}$ that are functions of the predetermined values of $P_{c \mid 0}, \bar{P}_{m d}$ and $\bar{P}_{f a}$. 


\section{A. Scenario 1: State prediction for $8 \mathrm{hrs}$}

During the measurement update period of the first $4 \mathrm{hrs}$, the range and angles measurements are used to estimate the position of the natural satellite, and then the states are predicted to the time of closest approach at 18 Feb 2019 12:00:00.000 UTC. A $P_{c \mid 0}$ value of 0.052075 is calculated as defined in Equation 6, for a hard body radius of 10 meters and assumed initial uncertainties. Figure 1, illustrates the progression of the simulated natural satellite and the OSIRIS-REx spacecraft over the total 12 hour period up to the time of closest approach.

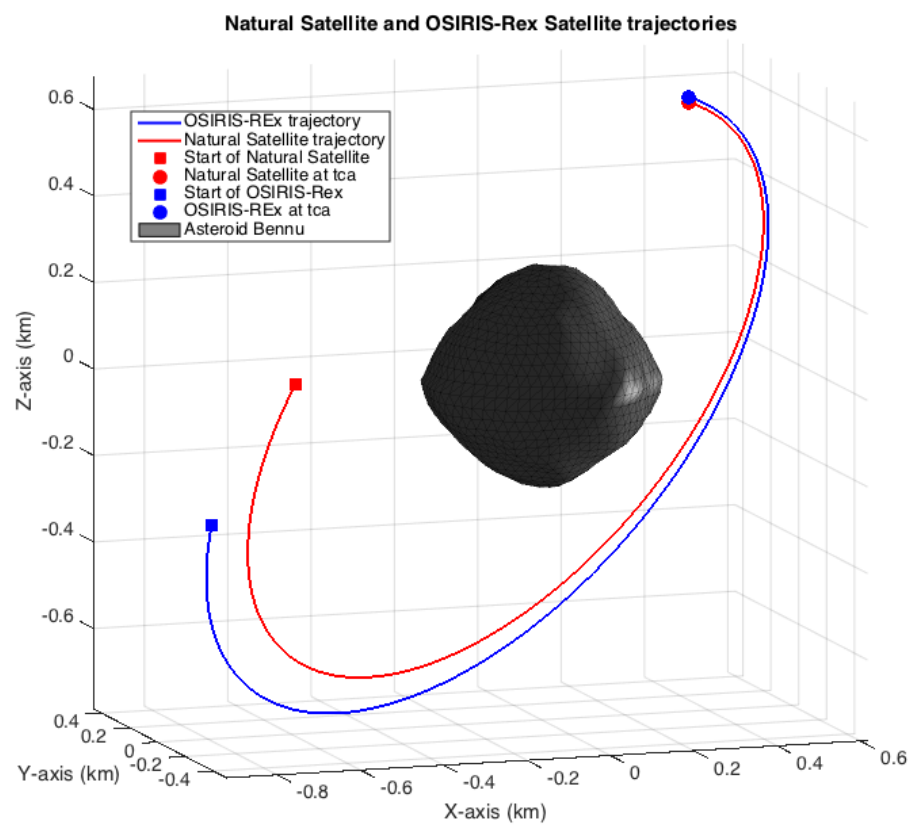

Figure 1. Relative position of OSIRIS-REx, True and Measured Natural Satellite positions towards closest approach

After the measurement updates, the $P_{c \mid k}$ (where $k=1,2, \ldots, 8 \mathrm{hrs}$ ) is calculated at each hour post the 4 hour measurements for the 500 Monte Carlo cases at the time of closest approach. The goal of calculating the $P_{c \mid k}$ at each hour, is to determine if a decision can be made on the point of closest approach before we reach $t_{c a}$. The decision to maneuver or not can be made based on the predetermined target false alarm, target missed detection rate, and the limits $\mathrm{A}$ and $\mathrm{B}$ values that are functions of the likelihood ratio.

In Table 3, a summary of the calculated parameters are presented for four case runs with mixed and matched targeted false alarm rates and missed detection rates of 0.2 and 0.01 . The alarm and dismissal limits show the boundaries for the $P_{c \mid k}$ values that will flag for a required maneuver or a dismissal (i.e. the conjunction is safe). The resulting parameter values for the four cases differ significantly for some of the terms, given the large differences in the defined target missed detection and false alarm rates.

In comparing the four cases with varied $\bar{P}_{f a}$ and $\bar{P}_{m d}$ values, we observe that the high $P_{c}^{\text {Alarm }}$ values of 0.844687 and 0.814638 are attributed to the low targeted false alarm rate $\left(\bar{P}_{f a}\right)$ value of 0.01 , that can be deduced from evaluating Equation 8 and Equation 11. This low targeted false alarm rate results into the low output of the number of sample cases that will trigger an alarm compared to the large numbers when the targeted false alarm rate used was 0.02. A low targeted missed detection also results in low dismissal limits as can also be seen in Figure 4 (b) and (c), where the solid black line has a much lower threshold compared to those of Figure 4 (a) and (d). This is evident in the number of sample cases that resulted to a "No Decision" output of 137 and 81 for cases (b) and (d) compared to 3 and 24 for cases (a) and (c). This means that the likelihood ratio is less than $\mathrm{A}$ and larger than $\mathrm{B}$, and if possible, more measurements should be taken to resolve this indecision. Moreover, in Figures 4, it can be seen that using a high targeted false alarm rate "allows" more sample cases to exceed the $P_{c}^{\text {Alarm }}$ trigger and at the same time induce more false alarm than true alarm sample cases. A low targeted false alarm rate value is a more conservative approach 
that limits false alarm events as was evident in the values for false alarms in Table 3 for the 0.01 targeted false alarm value.

Additionally, in the cases where the target false alarm rate of 0.2 was used, these cases resulted in a larger number of alarms triggered, however most of them were also false alarms compared to cases (b) and (d).

Table 3. Decision making computed parameters for 500 Monte Carlo runs (8 hrs post last measurement update)

\begin{tabular}{|c|c|c|c|c|}
\hline Parameter & (a) $\bar{P}_{f a_{0.2}}, \bar{P}_{m d_{0.2}}$ & (b) $\bar{P}_{f a_{0.01}}, \bar{P}_{m d_{0.01}}$ & (c) $\bar{P}_{f a_{0.2},}, \bar{P}_{m d_{0.01}}$ & (d) $\bar{P}_{f a_{0.01}}, \bar{P}_{m d_{0.2}}$ \\
\hline Alarm Limit $P_{c}^{\text {Alarm }}$ & 0.180155 & 0.844687 & 0.213794 & 0.814638 \\
\hline Dismissal Limit $P_{c}^{\text {Dismiss }}$ & 0.013548 & 0.000555 & 0.000686 & 0.010976 \\
\hline Alarms & 234 & 77 & 242 & 75 \\
\hline Dismissals & 263 & 286 & 234 & 344 \\
\hline Hits & 19 & 19 & 19 & 481 \\
\hline Misses & 481 & 481 & 17 & 11 \\
\hline True Alarms & 17 & 11 & 225 & 64 \\
\hline False Alarms & 217 & 66 & 232 & 341 \\
\hline True Dismissals & 261 & 284 & 2 & 3 \\
\hline False Dismissals & 2 & 2 & 24 & 81 \\
\hline No Decisions & 3 & 137 & $46.78 \%$ & $13.31 \%$ \\
\hline False Alarm Rate & $45.11 \%$ & $13.72 \%$ & $10.53 \%$ & $15.79 \%$ \\
\hline Missed Detection Rate & $10.53 \%$ & $10.53 \%$ & & \\
\hline
\end{tabular}

However, with significantly smaller targeted rates, it is possible to reduce the false alarm rate and the tradeoff would be a possible impact to the time it takes to reach a decision.

Another piece of insight in the decision making scheme would be the predictive uncertainty of the miss position vector. The position uncertainty shown in Table 4 demonstrates the measure of the proximity of the natural satellite to the spacecraft at the time of $t_{c a}$. It also shows that at the 4 th hour of prediction the miss distance at $t_{c} a$ is the smallest and in the situation that a decision may have not been made yet, this would definitely suppplement the decision making process to reduce any missed detections.

Table 4. Predictive position uncertainty at $t_{c a}$ over the $8 \mathrm{hrs}$ predictive period

\begin{tabular}{|c|c|c|}
\hline Variance in $\mathrm{x}\left(\mathrm{km}^{2}\right)$ at $t_{* \mid k}$ & Variance in y $\left(\mathrm{km}^{2}\right)$ at $t_{* \mid k}$ & Variance in z $\left(\mathrm{km}^{2}\right)$ at $t_{* \mid k}$ \\
\hline$\sigma_{x_{* \mid 1}}^{2}=0.0235$ & $\sigma_{y_{* \mid 1}}^{2}=0.0073$ & $\sigma_{z_{* \mid 1}}^{2}=0.0002$ \\
\hline$\sigma_{x_{* \mid 2}}^{2}=0.0003317$ & $\sigma_{y_{* \mid 2}}^{2}=0.0002548$ & $\sigma_{z_{* \mid 2}}^{2}=0.0000555$ \\
\hline$\sigma_{x_{* \mid 3}}^{2}=0.0001852$ & $\sigma_{y_{* \mid 3}}^{2}=0.0001093$ & $\sigma_{z_{* \mid 3}}^{2}=0.0000489$ \\
\hline$\sigma_{x_{* \mid 4}}^{2}=0.0001100$ & $\sigma_{y_{* \mid 4}}^{2}=0.0000755$ & $\sigma_{z_{* \mid 4}}^{2}=0.0000488$ \\
\hline$\sigma_{x_{* \mid 5}}^{2}=0.0013$ & $\sigma_{y_{* \mid 5}}^{2}=0.0043$ & $\sigma_{z_{* \mid 5}}^{2}=0.0012$ \\
\hline$\sigma_{x_{* \mid 6}}^{2}=0.0044$ & $\sigma_{y_{* \mid 6}}^{2}=0.0033$ & $\sigma_{z_{* \mid 6}}^{2}=0.0004$ \\
\hline$\sigma_{x_{* \mid 7}}^{2}=0.0005$ & $\sigma_{y_{* \mid 7}}^{2}=0.0045$ & $\sigma_{z_{* \mid 7}}^{2}=0.0004$ \\
\hline$\sigma_{x_{* \mid 8}}^{2}=0.0001$ & $\sigma_{y_{* \mid 8}}^{2}=0.0019$ & $\sigma_{z_{* \mid 8}}^{2}=0.0004$ \\
\hline
\end{tabular}

\section{B. Scenario 2: State prediction for $3 \mathrm{hrs}$}

During the measurement update period of the first $4 \mathrm{hrs}$, the range and angles measurements are used to estimate the position of the natural satellite, and then the states are predicted to the time of closest approach at 18 Feb 2019 07:00:00.000 UTC. A $P_{c \mid 0}$ value of 0.052075 is also calculated as defined in Equation 6, for a hard body radius of 10 meters. Figure 3 , illustrates the progression of the simulated natural satellite and the OSIRIS-REx spacecraft over the 7 hour period up to the time of closest approach.

For a set of varying targeted $\bar{P}_{f a}$ and a targeted $\bar{P}_{m d}$ values, we calculate the decision limits A and B and compare these values to the alarm and dismissal thresholds, similar to the first scenario. As shown in Figure 3, it is clear that the two objects are quickly in conjunction to one another. 


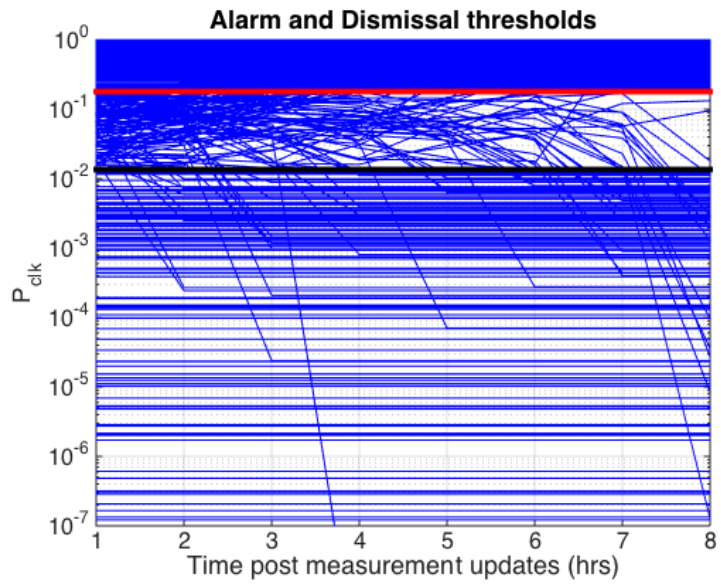

(a) $\bar{P}_{f a}=0.2$ and $\bar{P}_{m d}=0.2$.

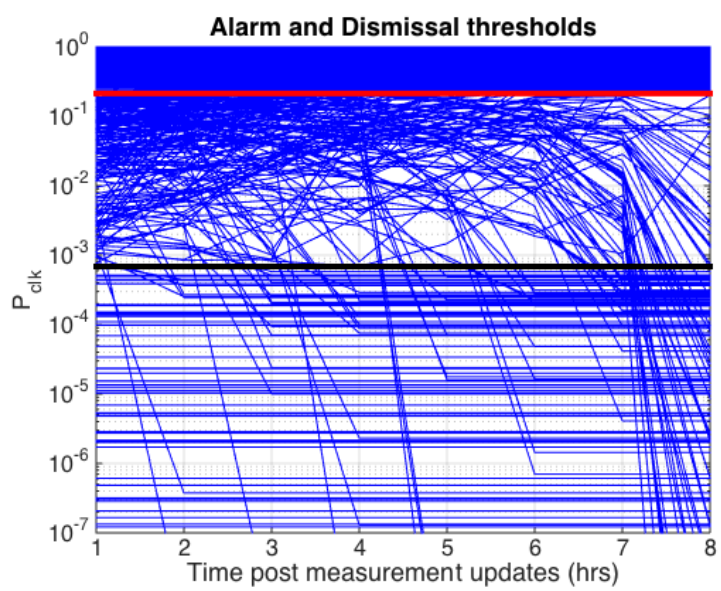

(c) $\bar{P}_{f a}=0.2$ and $\bar{P}_{m d}=0.01$

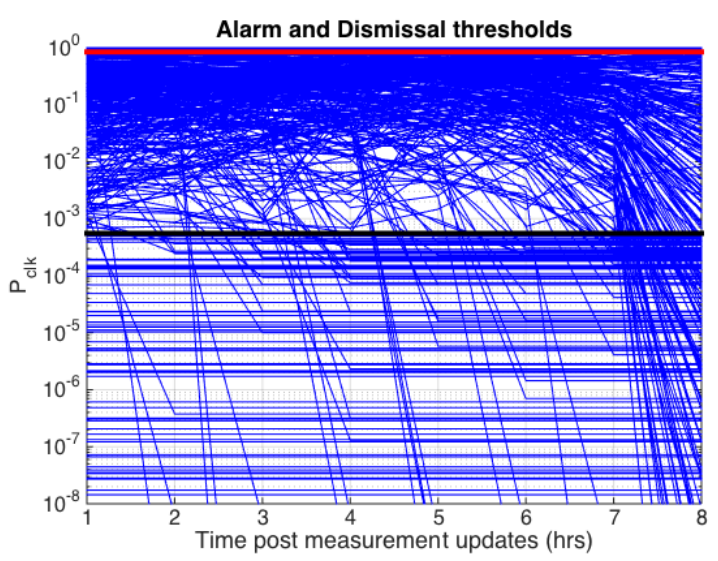

(b) $\bar{P}_{f a}=0.01$ and $\bar{P}_{m d}=0.01$

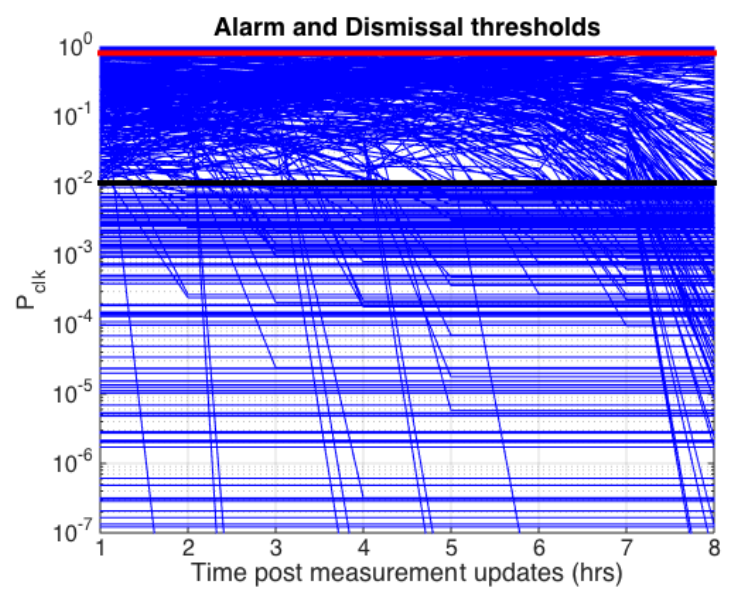

(d) $\bar{P}_{f a}=0.01$ and $\bar{P}_{m d}=0.2$

Figure 2. Cases (a) - (d); $P_{c \mid k}$ values for the next 8 hours. The red horizontal line is $P_{c}^{\text {Alarm }}$ and the black horizontal line is $P_{c}^{\text {Dismiss }}$. 


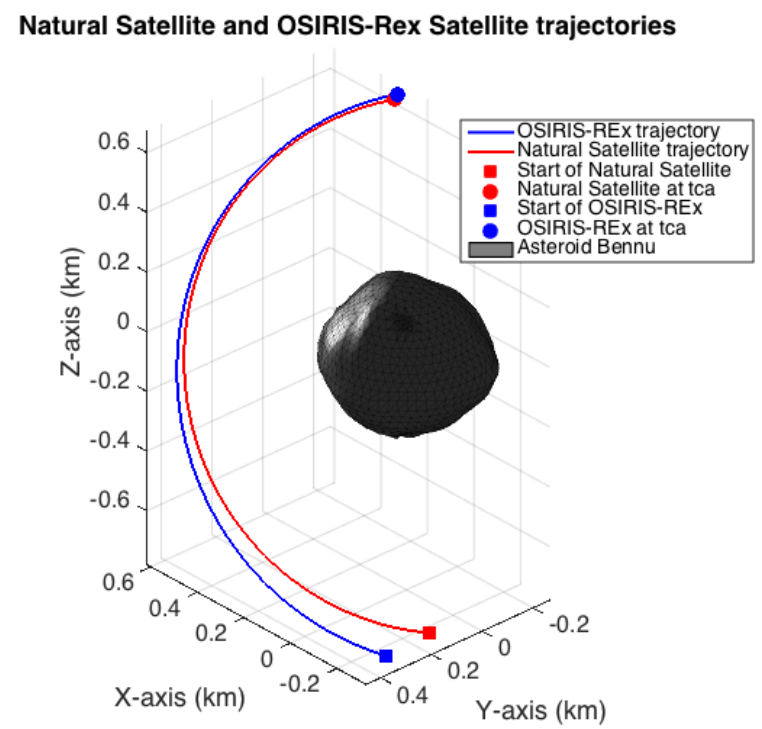

Figure 3. Relative position of OSIRIS-REx, True and Measured Natural Satellite positions towards closest approach

We want to determine whether the shorter prediction times affect the resulting decision making parameters and how do the varied targeted false alarm and missed detection rates perform under these new constraints. In Table 5, we see that most of the values are similar to Table 3, with the exception of the "No Decision" parameter. Given the short prediction period it is clear that the WSPRT may need to start ephemeris prediction much earlier for an improved decision making process. Comparing the false alarm rates for the targeted false alarm rates of 0.01 , we observe that the false alarm rates are slightly higher than those in Table 3.

The valuable insight obtained here, goes back to knowing how to balance the desired low missed detection rate and desired high false alarm rate outputs. Just having low targeted false alarm and missed detection rates does not always equate to a better decision making strategy. However, based on the simulated trajectories, the output true alarm values were about $2-3.6 \%$ of all 500 Monte Carlo cases and the variances in Table 5 , would imply that the right course of action would be to perform a maneuver. For the OSIRIS-REx mission, in the event that a natural satellites presents an impedance to proximity operations with stringent cadences, this is where valuable insight in assigning the targeted false alarm and targeted missed detection rates will be required. For such cases, where measurements cannot be readily available to track the natural satellites due to an ongoing science campaign that might also have geometric constraints, it may result in a need to have ample measurements and predicted ephemeris for the natural satellite before such campaigns.

\section{Conclusions and Future Work}

This was a preliminary study in determining how a conjunction analysis and decision making algorithm of a simulated natural satellite and the OSIRIS-REx spacecraft could be implemented. Given a subset of good sporadic measurement updates, a relatively accurate predicted trajectory can be generated for an accurate decision making process along with successive $P_{c \mid k}$ values and well designed $P_{c}^{\text {Alarm }}$ and $P_{c}^{\text {Dismiss }}$ values. The future work would encompass the use of a representative probability density function along with a myriad of targeted false alarm and missed detection rates that would better complement the OSIRIS-REx mission's collision avoidance decision making strategy. The decision making strategy will highly depend on the time constraints based on the cadence of proximity operations in a particular phase of operations as well as the available fuel budget for implementing a collision avoidance maneuver. Both these constraints will be further pursued in more specific phases and cadences of the OSIRIS-REx proximity operations. 


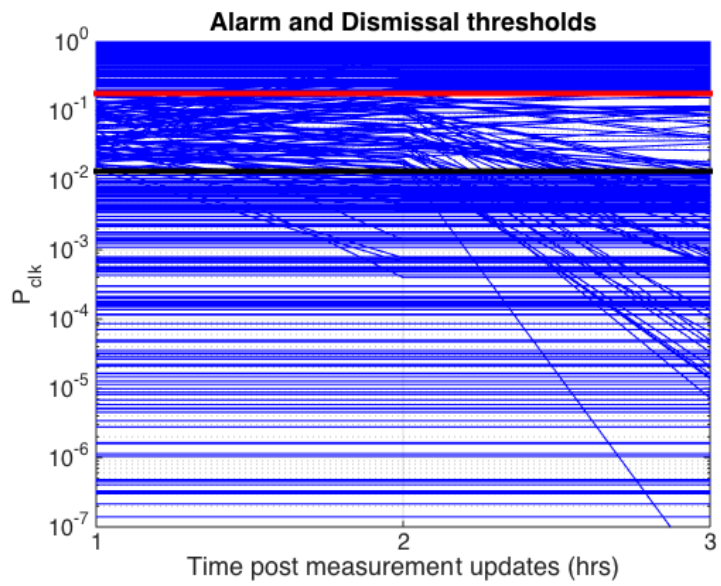

(a) $\bar{P}_{f a}=0.2$ and $\bar{P}_{m d}=0.2$.

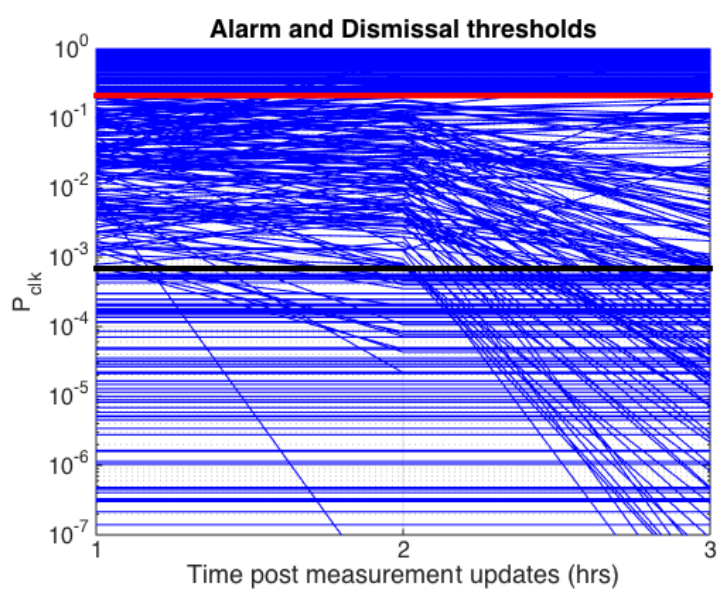

(c) $\bar{P}_{f a}=0.2$ and $\bar{P}_{m d}=0.01$

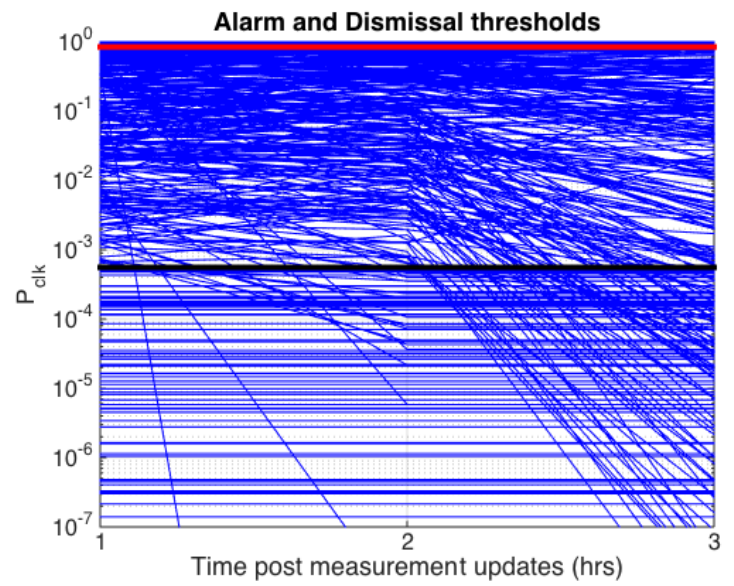

(b) $\bar{P}_{f a}=0.01$ and $\bar{P}_{m d}=0.01$

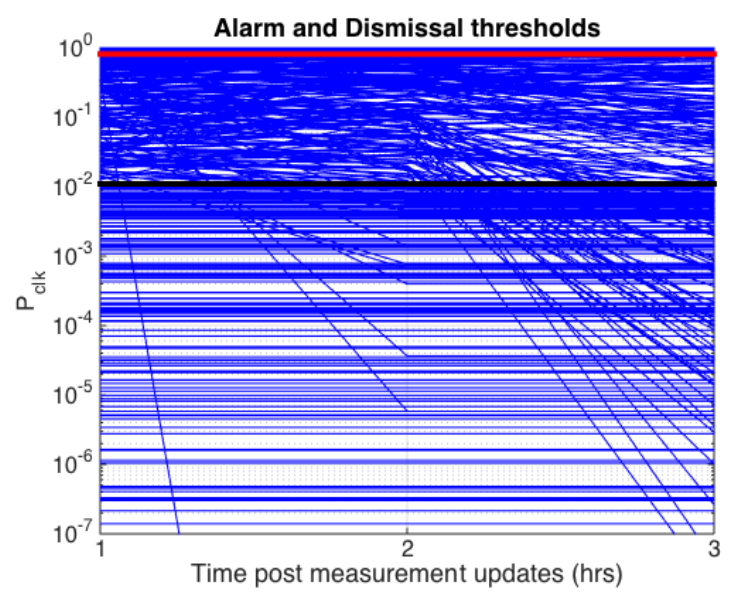

(d) $\bar{P}_{f a}=0.01$ and $\bar{P}_{m d}=0.2$

Figure 4. Cases (a) - (d); $P_{c \mid k}$ values for the next 3 hours. The red horizontal line is $P_{c}^{\text {Alarm }}$ and the black horizontal line is $P_{c}^{\text {Dismiss }}$.

Table 5. Decision making computed parameters for 500 Monte Carlo runs (3 hrs post last measurement update)

\begin{tabular}{|c|c|c|c|c|}
\hline Parameter & (a) $\bar{P}_{f a_{0.2},}, \bar{P}_{m d_{0.2}}$ & (b) $\bar{P}_{f a_{0.01}}, \bar{P}_{m d_{0.01}}$ & (c) $\bar{P}_{f a_{0.2}}, \bar{P}_{m d_{0.01}}$ & (d) $\bar{P}_{f a_{0.01}}, \bar{P}_{m d_{0.2}}$ \\
\hline Alarm Limit $\left(P_{c}^{\text {Alarm }}\right)$ & 0.180155 & 0.844687 & 0.213794 & 0.814638 \\
\hline Dismissal Limit $\left(P_{c}^{\text {Dismiss }}\right)$ & 0.013548 & 0.000555 & 0.000686 & 0.010976 \\
\hline Alarms & 198 & 91 & 195 & 96 \\
\hline Dismissals & 273 & 236 & 229 & 282 \\
\hline Hits & 19 & 19 & 19 & 19 \\
\hline Misses & 481 & 481 & 481 & 181 \\
\hline True Alarms & 18 & 14 & 17 & 81 \\
\hline False Alarms & 180 & 77 & 228 & 281 \\
\hline True Dismissals & 272 & 235 & 1 & 1 \\
\hline False Dismissals & 1 & 1 & 76 & 122 \\
\hline No Decisions & 29 & 173 & $36.80 \%$ & $16.84 \%$ \\
\hline False Alarm Rate & $37.42 \%$ & $16.01 \%$ & $5.26 \%$ & $5.26 \%$ \\
\hline Missed Detection Rate & $5.26 \%$ & $5.26 \%$ & & \\
\hline
\end{tabular}


Table 6. Predictive position covariance at $t_{c a}$ over the $3 \mathrm{hrs}$ predictive period

\begin{tabular}{|c|c|c|}
\hline Variance in x $\left(\mathrm{km}^{2}\right)$ at $t_{* \mid k}$ & Variance in $\mathrm{y}\left(\mathrm{km}^{2}\right)$ at $t_{* \mid k}$ & Variance in $\mathrm{z}\left(\mathrm{km}^{2}\right)$ at $t_{* \mid k}$ \\
\hline$\sigma_{x_{* \mid 1}}^{2}=0.0955$ & $\sigma_{y_{* \mid 1}}^{2}=0.0048$ & $\sigma_{z_{* \mid 1}}^{2}=0.0004$ \\
\hline$\sigma_{x_{* \mid 2}}^{2}=0.0026$ & $\sigma_{y_{* \mid 2}}^{2}=0.0019$ & $\sigma_{z_{* \mid 2}}^{2}=0.0004$ \\
\hline$\sigma_{x_{* \mid 3}}^{2}=0.0005$ & $\sigma_{y_{* \mid 3}}^{2}=0.0011$ & $\sigma_{z_{* \mid 3}}^{2}=0.0005$ \\
\hline
\end{tabular}

\section{Acknowledgements}

The authors would like to thank Kevin Berry of the Navigation and Mission Design Branch at NASA Goddard Space Flight Center for reviewing this paper.

\section{References}

${ }^{1}$ Chesley, S. R. et al., "Orbit and bulk density of the OSIRIS-REx target Asteroid (101955) Bennu," Icarus, Vol. 235, 2014 , pp. 5-22.

${ }^{2}$ Nolan, M. C., "Shape model and surface properties of the OSIRIS-REx target Asteroid (101955) Bennu from radar and lightcurve observations," Icarus, Vol. 226, 2013, pp. 629-640.

${ }^{3}$ Margot, J. et al., "Binary asteroids in the Near-Earth object Population," Science, Vol. 296, No. 5572, 2002.

${ }^{4}$ Wikipedia, "Minor-planet moon," 2015.

${ }^{5}$ Britt, D. and Consolmagno, G., "Stony meteorite porosities and densities: A review of the data through 2001," Meteoritics and Planetary Science, Vol. 38, No. 8, August 2003, pp. 1161-1180.

${ }^{6}$ Kessler, D., Reynolds, R., and Anz-Meador, P., "Orbital Debris Environment for Space Craft Designed to Operate in Low Earth Orbit," Tech. rep., JSC TM 100-471, April 1989.

${ }^{7}$ Foster, J. L. and Estes, H. S., "A Parametric Analysis of Orbital Debris Collision Probability and Maneuver Rate for Space Vehicles," Tech. rep., Mission Operations Directorate (JSC), 1992.

${ }^{8}$ Carpenter, J. and Markley, F., "Wald Sequential Probability Ratio Test for Space Object Conjunction Assessment," Journal of Guidance, Control and Dynamics, Vol. 37, No. 5, 2014, pp. 1385-1396.

${ }^{9}$ Patera, R. P., "Satellite Collision Probability for Nonlinear Relative Motion," Journal of Guidance, Control and Dynamics, Vol. 26, No. 3, 2003, pp. 728-733.

10

${ }^{11}$ G.G.Wawrzyniak, Carpenter, J., Mattern, D., Wiliams, T., and Ottenstein, N., "Conjunction Assessment Concept of Operations for the Magnetospheric Mulit-Scale (MMS) Mission," Advances in Astronautical Sciences, Vol. 150, 2013, pp. $181-200$.

${ }^{12}$ Carpenter, J., Markley, F., and Gold, D., "Sequential Probability Ratio Test for Collision avoidance maneuver decisions," The Journal of the Astronautical Sciences, Vol. 59, No. 1, January-June 2012, pp. 273-286.

${ }^{13}$ Wald, A., Sequential Analysis, Dover, New York, 2004. 